PREPARED FOR THE U.S. DEPARTMENT OF ENERGY, UNDER CONTRACT DE-AC02-76CH03073

PPPL-3946

PPPL-3946

UC-70

Variational Formulation
of the Gardner's Restacking Algorithm

by

I.Y. Dodin and N.J. Fisch

April 2004

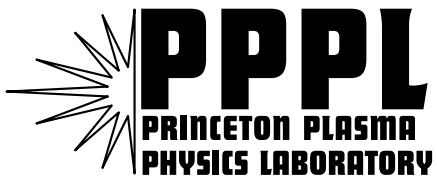

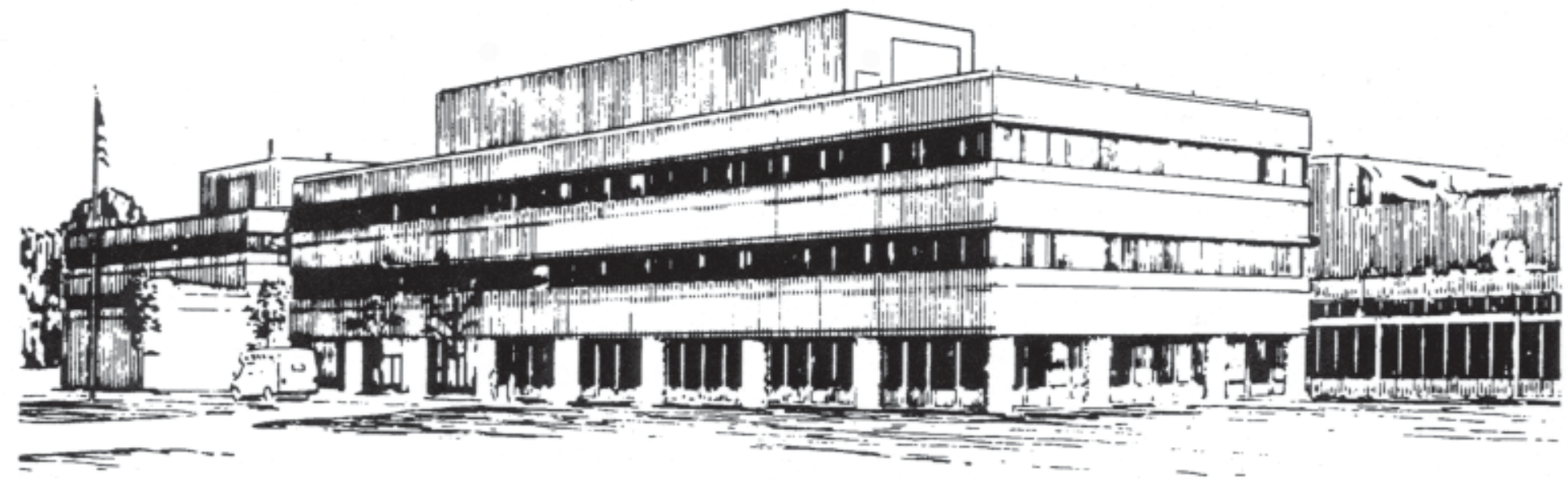

PRINCETON PLASMA PHYSICS LABORATORY PRINCETON UNIVERSITY, PRINCETON, NEW JERSEY 


\section{PPPL Reports Disclaimer}

This report was prepared as an account of work sponsored by an agency of the United States Government. Neither the United States Government nor any agency thereof, nor any of their employees, makes any warranty, express or implied, or assumes any legal liability or responsibility for the accuracy, completeness, or usefulness of any information, apparatus, product, or process disclosed, or represents that its use would not infringe privately owned rights. Reference herein to any specific commercial product, process, or service by trade name, trademark, manufacturer, or otherwise, does not necessarily constitute or imply its endorsement, recommendation, or favoring by the United States Government or any agency thereof. The views and opinions of authors expressed herein do not necessarily state or reflect those of the United States Government or any agency thereof.

\section{Availability}

This report is posted on the U.S. Department of Energy's Princeton Plasma Physics Laboratory Publications and Reports web site in Fiscal Year 2004. The home page for PPPL Reports and Publications is: http://www.pppl.gov/pub_report/

DOE and DOE Contractors can obtain copies of this report from:

U.S. Department of Energy

Office of Scientific and Technical Information

DOE Technical Information Services (DTIS)

P.O. Box 62

Oak Ridge, TN 37831

Telephone: (865) 576-8401

Fax: (865) 576-5728

Email: reports@adonis.osti.gov

This report is available to the general public from:

National Technical Information Service

U.S. Department of Commerce

5285 Port Royal Road

Springfield, VA 22161

Telephone: $1-800-553-6847$ or

(703) $605-6000$

Fax: (703) 321-8547

Internet: http://www.ntis.gov/ordering.htm 


\title{
Variational formulation of the Gardner's restacking algorithm
}

\author{
I.Y. Dodin and N.J. Fisch \\ Princeton Plasma Physics Laboratory, Princeton, NJ 08543
}

\begin{abstract}
The incompressibility of the phase flow of Hamiltonian wave-plasma interactions restrains the class of realizable wave-driven transformations of the particle distribution. After the interaction, the distribution remains composed of the original phase-space elements, or local densities, which are only rearranged ("restacked") by the wave. A variational formalism is developed to study the corresponding limitations on the energy and momentum transfer. A case of particular interest is a toroidal plasma immersed in a dc magnetic field. The restacking algorithm by Gardner [Phys. Fluids 6, 839 (1963)] is formulated precisely. The minimum energy state for a plasma with a given current is determined.
\end{abstract}

\section{INTRODUCTION}

The incompressibility of the phase flow of Hamiltonian wave-plasma interactions restrains the class of realizable wave-driven transformations of the particle distribution [1-6]. In particular, this fact determines the existence of what can be called the "plasma ground state" for a given one-particle distribution $f_{1}$. By ground state, we mean such a distribution of particles $f_{2}$, which minimizes the total plasma energy on the manifold of all Hamiltonian transformations $f_{1} \rightarrow f_{2}$.

As reported in the pioneering paper by Gardner [1], the ground state plasma energy $W_{\text {min }}$ is generally nonzero, which can be explained as follows. Suppose that a bounded plasma particles having the initial phase-space distribution $f_{1}$ are introduced into an electromagnetic field for a limited time, which eventually results in bringing the plasma to some final state $f_{2}$. Imagine that we partition the plasma phase space into small cells of equal volume $\Delta \Gamma_{i}=\Delta \Gamma$, and to each cell attach a certain value of the distribution function $f\left(\Gamma_{i}\right)$. As the number of cells that have a given value of $f$ is conserved throughout the interaction (as follows from the Liouville theorem), the distribution $f_{2}$ may not be arbitrary, but rather will represent a result of reordering ("restacking") of the original phase-space elements $\Delta \Gamma_{i}$, regardless of the spatial and temporal structure of the external fields. Alternatively, this fact can be expressed as conservation of the so-called Casimir invariants, which determine the distribution of the values $f\left(\Gamma_{i}\right)(s e e, e . g .$, Ref. [7]) and whose existence is an intrinsic property of any Hamiltonian system.

The plasma ground state will correspond to the distribution $f_{2}$, such that the elements $\Delta \Gamma_{i}$ with larger $f\left(\Gamma_{i}\right)$ occupy the states with lower particle energy $\mathcal{E}$. In a bounded plasma, only a finite phase volume is allotted to the states with $\mathcal{E}$ below a given value. Hence, from incompressibility of the phase flow, it follows that after the interaction the plasma will be left with the total energy $W \geq W_{\min }$, where $W_{\min }$ is a nonzero quantity defined as the minimum of $W$ over all possible ways of restacking the elements $\Delta \Gamma_{i}$.

While chopping phase space into discrete elements is pictorial, it is fairly artificial in case of a continuous function $f_{1}$. Hence, solving the "restacking problem" must be possible in terms of a differential formulation. The purpose 
of the present paper is to derive such a formulation and apply it to a number of cases of interest, not previously considered.

The paper is organized as follows: In Sec. II, we generalize the Gardner's problem by putting it into a variational form for an abstract dynamical system. We determine the condition under which a Hamiltonian transformation of the system phase space yields the maximum or minimum of a given functional (such as the plasma energy in Ref. [1]). In the framework of this formalism, we reproduce the results given in Ref. [1] and, in Sec. III, solve a similar, yet different problem of finding the minimum energy state at given plasma current. In Sec. IV, we apply our formalism to magnetized toroidal plasmas and derive a reduced variational principle. In Sec. V, we summarize our main ideas.

\section{VARIATIONAL FORMALISM}

Let us first restate the Gardner's problem in its original form [1]. Suppose that a bounded plasma with the initial distribution $f\left(\mathbf{r}_{1}, \mathbf{p}_{1}\right)$ is introduced into external fields for a limited time, which eventually results in bringing the plasma to some final state $f\left(\mathbf{r}_{2}, \mathbf{p}_{2}\right)$. The particle distribution is conserved: $f\left(\Gamma_{2}\right)=f\left(\Gamma_{1}\right)$, where $\Gamma_{2} \equiv\left(\mathbf{r}_{2}, \mathbf{p}_{2}\right)$ is a single-valued reversible function of $\Gamma_{1} \equiv\left(\mathbf{r}_{1}, \mathbf{p}_{1}\right)$. Thus, the total energy left inside the plasma after the interaction equals

$$
W=\int \mathcal{E}\left(\Gamma_{2}\right) f\left(\Gamma_{1}\right) d \Gamma
$$

where $\mathcal{E}$ is the individual particle energy, and where we made use of phase space conservation: $d \Gamma \equiv d \Gamma_{1}=d \Gamma_{2}$, $d \Gamma_{i} \equiv d^{3} r_{i} d^{3} p_{i}$. Suppose that the particles initially occupy a nonzero phase volume. In a bounded plasma, only a finite phase volume is allotted to the states with $\mathcal{E}\left(\Gamma_{2}\right)$ below a given value. Hence, from incompressibility of the phase flow it follows that after the interaction the plasma will be left with the total energy $W \geq W_{\text {min }}$, where

$$
W_{\min }=\min _{\Gamma_{1} \rightarrow \Gamma_{2}} \int \mathcal{E}\left(\Gamma_{2}\right) f\left(\Gamma_{1}\right) d \Gamma
$$

is a nonzero quantity defined as the minimum of $W$ over all possible Hamiltonian (canonical) phase-space transformations $\left(\mathbf{r}_{1}, \mathbf{p}_{1}\right) \rightarrow\left(\mathbf{r}_{2}, \mathbf{p}_{2}\right)$. Hence, determination of $W_{\min }$ can be considered as a variational problem of searching for the canonical transformation $\Gamma_{1} \rightarrow \Gamma_{2}$, which minimizes the functional (1).

Treated like that, the Gardner's problem yields a natural generalization as follows. Suppose one is given a function $\phi\left(\Gamma_{1}\right)$ defined in a $2 N$-dimensional phase space $\Gamma_{1}$. Suppose also that $\Gamma_{1} \equiv(\mathbf{q}, \mathbf{p})$ undergoes Hamiltonian evolution into some phase space $\Gamma_{2} \equiv(\mathbf{Q}, \mathbf{P})$. The generalized Gardner's problem then consists of determining the connection between $\Gamma_{1}$ and $\Gamma_{2}$, which provides the minimum or the maximum of the functional

$$
G=\int \psi\left(\Gamma_{2}\right) \phi\left(\Gamma_{1}\right) d \Gamma
$$

where $\Gamma_{2}$ is considered a function of $\Gamma_{1}$ (or vice versa), $\psi$ is a known function of $\Gamma_{2}$, and $d \Gamma \equiv d \Gamma_{1}=d \Gamma_{2}$ is a phase space element

$$
d \Gamma \equiv d^{N} q d^{N} p=d^{N} Q d^{N} P
$$


conserved by the canonical transformation.

Suppose $\Gamma_{1} \rightarrow \Gamma_{2}$ is an extremizing transformation and consider a small canonical transformation $\Gamma_{2} \rightarrow \Gamma_{2}^{\prime}$ determined by an arbitrary trial Hamiltonian $\mathcal{H}$. Since $\phi$ is defined as a given function of the initial variables, which remain unchanged by the transformation, one gets

$$
\Delta G=\int \Delta \psi\left(\Gamma_{2}\right) \phi\left(\Gamma_{1}\right) d \Gamma_{1}
$$

The change of $\psi, \Delta \psi=\psi\left(\Gamma_{2}^{\prime}\right)-\psi\left(\Gamma_{2}\right)$, can be expressed as

$$
\Delta \psi \approx \frac{\partial \psi}{\partial \mathbf{Q}} \cdot \Delta \mathbf{Q}+\frac{\partial \psi}{\partial \mathbf{P}} \cdot \Delta \mathbf{P}+\frac{1}{2} \Delta \mathbf{Q} \cdot \frac{\partial^{2} \psi}{\partial \mathbf{Q} \partial \mathbf{Q}} \cdot \Delta \mathbf{Q}++\Delta \mathbf{Q} \cdot \frac{\partial^{2} \psi}{\partial \mathbf{Q} \partial \mathbf{P}} \cdot \Delta \mathbf{P}+\frac{1}{2} \Delta \mathbf{P} \cdot \frac{\partial^{2} \psi}{\partial \mathbf{P} \partial \mathbf{P}} \cdot \Delta \mathbf{P},
$$

where the dot product stands for summation over repeating indices. The changes of $\Delta \mathbf{Q}$ and $\Delta \mathbf{P}$ are derivable from canonical equations and can be put in the form

$$
\begin{aligned}
& \Delta \mathbf{Q}=\Delta t \frac{\partial \mathcal{H}}{\partial \mathbf{P}}+\frac{\Delta t^{2}}{2}\left\{\mathcal{H}, \frac{\partial \mathcal{H}}{\partial \mathbf{P}}\right\}+o\left(\Delta t^{2}\right), \\
& \Delta \mathbf{P}=-\Delta t \frac{\partial \mathcal{H}}{\partial \mathbf{Q}}-\frac{\Delta t^{2}}{2}\left\{\mathcal{H}, \frac{\partial \mathcal{H}}{\partial \mathbf{Q}}\right\}+o\left(\Delta t^{2}\right),
\end{aligned}
$$

where $\Delta t$ is the time interval, on which the evolution generated by $\mathcal{H}$ is considered, and $\{\cdot, \cdot\}$ stands for Poisson brackets

$$
\{f, g\} \equiv \frac{\partial f}{\partial \mathbf{P}} \cdot \frac{\partial g}{\partial \mathbf{Q}}-\frac{\partial f}{\partial \mathbf{Q}} \cdot \frac{\partial g}{\partial \mathbf{P}} .
$$

Substituting the above equations into Eq. (5) and integrating by parts, one gets that

$$
\begin{aligned}
& \Delta G=\delta G+\delta^{2} G+o\left(\Delta t^{2}\right), \\
& \delta G=\Delta t \int\{\psi, \phi\} \mathcal{H} d \Gamma, \\
& \delta^{2} G=-\frac{\Delta t^{2}}{2} \int\{\psi, \mathcal{H}\}\{\phi, \mathcal{H}\} d \Gamma,
\end{aligned}
$$

assuming that the surface integrals are equal to zero. (In case if $\psi$ or $\phi$ stands for a distribution function, the surface integrals vanish, e.g., if the system is localized within a finite phase volume.) Because of the invariance of Poisson brackets, the above expressions equally apply to any space $\Gamma$ canonically obtainable from $\Gamma_{1}$ or $\Gamma_{2}$ ( $\Gamma$ may also coincide with one of the two), if the functions are understood as

$$
\phi=\phi\left(\Gamma_{1}(\Gamma)\right), \quad \psi=\psi\left(\Gamma_{2}(\Gamma)\right)
$$

From Eq. (9) it follows that the necessary condition for an extremizer, that is $\delta G=0$ for an arbitrary $\mathcal{H}$, can be put in the form

$$
\{\psi, \phi\}=0
$$

In turn, the minimum and maximum of $G$ are realized when $\delta^{2} G$ has a definite sign regardless of $\mathcal{H}$. Noting that

$$
\delta \psi=\Delta t\{\mathcal{H}, \psi\}, \quad \delta \phi=\Delta t\{\mathcal{H}, \phi\}
$$


one can rewrite the expression for $\delta^{2} G$ as

$$
\delta^{2} G=-\frac{1}{2} \int \delta \psi \delta \phi d \Gamma
$$

From Eq. (13) it can be concluded that the minimum of $G$ is achieved if

$$
\phi=\phi(\psi), \quad \text { or } \quad \psi=\psi(\phi)
$$

is a single-valued monotonically decreasing function; the maximum of $G$ corresponds to a single-valued monotonically increasing function (14). (Note also that Eq. (14) automatically satisfies Eq. (11).)

The function (14) can be determined using the phase-space conservation imposed by the Liouville theorem. With the density of states $\Omega$ defined for an arbitrary function $\xi(\Gamma)$ according to

$$
\Omega(\tilde{\xi}) \equiv \int \delta(\tilde{\xi}-\xi(\Gamma)) d \Gamma
$$

the phase-space conservation requires that

$$
\left|\frac{d \phi}{d \psi}\right|=\frac{\Omega(\psi)}{\Omega(\phi)}, \quad \phi\left(\psi_{0}\right)=\phi_{0} .
$$

The sign of the derivative and the constant of integration must be chosen correspondingly, depending on whether the maximum or the minimum of $G$ is required: taking $d \phi / d \psi \geq 0$ with $\phi\left(\psi_{\max }\right)=\phi_{\max }$ yields the maximizer, while $d \phi / d \psi \leq 0$ with $\phi\left(\psi_{\max }\right)=\phi_{\min }$ corresponds to the minimizer.

If $\phi\left(\Gamma_{1}\right)$ and $\psi\left(\Gamma_{2}\right)$ are continuous functions, then the transformation, which yields the absolute minimum or the absolute maximum of $G$, is at least piecewise continuous. In this case, the differential formulation as presented here is more natural than the "Gardner's restacking algorithm" described in Ref. [3]. Making use of the differential formulation in certain cases can yield an analytical solution of the restacking problem or, at least, allow a solution by quadratures, hence simplifying the numerical procedure of finding the extremized functional value. On the other hand, our results can also be formulated in terms of reordering of discrete phase space elements as follows: To obtain an extremizing transformation, first, chop the phase space $\Gamma$ into differentially small elements with equal volume $d \Gamma^{(i)}=d \Gamma$, each confined between the neighboring isosurfaces of $\phi$. Numerate the elements in ascending order with respect to $\phi\left(\Gamma^{(i)}\right)$. Then prepare the new "sites" for these elements - the phase space bins of the same volume, $d \tilde{\Gamma}^{(j)}=d \Gamma$, each located between the neighboring isosurfaces of $\psi$. Numerate them in ascending order with respect to $\psi\left(\tilde{\Gamma}^{(j)}\right)$ and allocate $\Gamma^{(i)}$ at $\tilde{\Gamma}^{(j)}$. The maximizing transformation requires that $i(j)$ be an increasing function, while the minimizing transformation requires that $i(j)$ be decreasing.

Let us apply the obtained results to reproduce the solution of the original Gardner's problem. To put the energy functional (1) into the form (3), take

$$
\phi\left(\Gamma_{1}\right)=f\left(\mathbf{r}_{1}, \mathbf{p}_{1}\right), \quad \psi\left(\Gamma_{2}\right)=\mathcal{E}_{2}\left(\mathbf{r}_{2}, \mathbf{p}_{2}\right)
$$

If $\mathcal{E}_{2}$ includes only the kinetic energy of a particle $\left(\mathcal{E}_{2}=p_{2}^{2} / 2 m\right)$, then the final particle distribution $f\left(\mathbf{r}_{2}, \mathbf{p}_{2}\right)$ corresponding to the minimum plasma energy cannot depend on $\mathbf{r}_{2}$, as follows already from Eq. (11). From the 
subsequent arguments, one gets that the final distribution must be a single-valued decreasing function of $\mathcal{E}_{2}$ and satisfy the equation

$$
\frac{d f}{d \mathcal{E}_{2}}=-\frac{\Omega\left(\mathcal{E}_{2}\right)}{\Omega(f)}
$$

where $f(0)=\max f\left(\mathbf{r}_{1}, \mathbf{p}_{1}\right)$. The density of states $\Omega(f)$ can be calculated knowing the initial distribution $f\left(\mathbf{r}_{1}, \mathbf{p}_{1}\right)$, and $\Omega\left(\mathcal{E}_{2}\right)$ is given by

$$
\Omega\left(\mathcal{E}_{2}\right)=4 \pi m \sqrt{2 m \mathcal{E}_{2}}
$$

The same analysis, including Eq. (18), applies if $\mathcal{E}_{2}$ contains also a potential energy of a particle in a static background field $U$,

$$
\mathcal{E}_{2}\left(\mathbf{r}_{2}, \mathbf{p}_{2}\right)=\frac{p_{2}^{2}}{2 m}+U\left(\mathbf{r}_{2}\right)
$$

if the density of states $\Omega\left(\mathcal{E}_{2}\right)$ is modified correspondingly. Hence, one can see that the results of Ref. [1] can be naturally obtained in the framework of the proposed formalism. Yet, the latter also yields other results of interest, as we show in the next sections.

\section{CONDITIONAL EXTREMUM}

The formalism presented in Sec. II yields a natural generalization to the case of a conditional restacking problem. Consider finding an extremum of the functional (3) under the condition

$$
\mathbf{R}=\mathbf{R}^{(0)}, \quad \mathbf{R}=\left\{R_{i} \mid i=1 . . K\right\}
$$

where

$$
R_{i}=\int \tilde{\psi}_{i}\left(\Gamma_{2}\right) \tilde{\phi}_{i}\left(\Gamma_{1}\right) d \Gamma
$$

assuming that $\tilde{\psi}_{i}\left(\Gamma_{2}\right), \tilde{\phi}_{i}\left(\Gamma_{1}\right)$ are given functions, and $\mathbf{R}^{(0)}=\left\{R_{i}^{(0)} \mid i=1 . . K\right\}$ is a set of constants. Conditional extrema of $G$ are realized at unconditional extremizers of the functional

$$
\tilde{G}=G+\lambda \cdot \mathbf{R}
$$

where $\boldsymbol{\lambda}=\left\{\lambda_{i} \mid i=1 . . K\right\}$ are indefinite Lagrange multipliers to be found. As seen from the previous analysis, the extrema of $\tilde{G}$ are realized under the condition

$$
\{\psi, \phi\}+\sum_{i=1}^{K} \lambda_{i}\left\{\tilde{\psi}_{i}, \tilde{\phi}_{i}\right\}=0 .
$$

If $\tilde{\phi}_{i}$ are all equal to $\phi$ (alternatively, all $\tilde{\psi}$ may be equal to $\psi$ ), Eq. (24) is simplified:

$$
\{\Psi, \phi\}=0
$$


where $\Psi=\psi+\boldsymbol{\lambda} \cdot \tilde{\boldsymbol{\psi}}$. Eq. (25) has the form of Eq. (11) and hence can be solved by the method proposed in Sec. II, with $\lambda_{i}$ to be determined from Eq. (21).

An illustrative example of how this formalism can be applied is the problem of finding (again, assuming phase-space conservation) the minimum energy state of a plasma with a given current. The problem has a definite practical value, as its solution determines how much energy is required for generating a given amount of plasma current. (Note, however, that this is not the problem that determines the "efficiency" of maintaining a current, since the maximum efficiency may not occur for the minimum energy distribution [8].) To get the minimum energy current, consider the frame of reference moving with the velocity $\mathbf{v}_{0}=\mathbf{j} / e n$, where $\mathbf{j}$ is the current density, $e$ is the charge of an individual particle, and $n$ is the particle number density. Solve the unconditional energy minimization problem for the new frame, as shown in Sec. II. The absolute minimum of the total particle energy is achieved at an isotropic distribution, which carries no current. On the other hand, in the moving frame, the current density must be zero by definition. Thus, the solution of the unconditional problem in the new frame satisfies the requirements of the original conditional problem. Hence, the minimum energy state at given current is realized at particle distribution isotropic and monotonically decreasing with energy in the frame of reference where the net current is zero.

Note that the same result can be obtained formally as follows. Consider the functional

$$
\tilde{G}=W+\boldsymbol{\lambda} \cdot \mathbf{j}
$$

where the plasma current, assuming given initial distribution $f\left(\mathbf{p}_{1}\right)$, equals

$$
\mathbf{j}=e \int \mathbf{v}_{2} f\left(\mathbf{p}_{1}\right) d^{3} p_{1}
$$

Rewrite Eq. (26) as

$$
\tilde{G}=\int \frac{\left(\mathbf{p}_{2}+\lambda e\right)^{2}}{2 m} f\left(\mathbf{p}_{1}\right) d^{3} p_{1}-\frac{\lambda^{2} e^{2}}{2 m}
$$

where the value of the second term is fixed, and take

$$
\phi\left(\Gamma_{1}\right)=f\left(\mathbf{p}_{1}\right), \quad \Psi\left(\Gamma_{2}\right)=\frac{\left(\mathbf{p}_{2}+\lambda e\right)^{2}}{2 m} .
$$

Hence, one can see that, to minimize $\tilde{G}$, the particle distribution must become a function of energy in the frame of reference moving with $\mathbf{v}_{0}=-\boldsymbol{\lambda} / m e$, and thus

$$
\tilde{G}_{\min }=W_{\min }^{\prime}-\frac{\lambda^{2} e^{2}}{2 m}
$$

Here $W_{\min }^{\prime}$ is the minimized energy in the moving frame, where the particle distribution must be isotropic (as follows from Sec. II), i.e. carry no current. The total current then equals en $\mathbf{v}_{0}$, hence

$$
\boldsymbol{\lambda}=-m \mathbf{j} / n e^{2} .
$$

On the other hand, by definition,

$$
\tilde{G}_{\min }=W_{\min }+\boldsymbol{\lambda} \cdot \mathbf{j} .
$$


Using Eqs. (30), (31), (32), one has

$$
W_{\min }=\frac{m j^{2}}{2 n e^{2}}+W_{\min }^{\prime}
$$

where the first term represents the energy of the average flow, while the second term stands for the minimum thermal energy of the original distribution $f\left(\mathbf{p}_{1}\right)$, which cannot be reduced further by Hamiltonian transformations of the original particle distribution.

\section{RESTACKING ALGORITHM FOR MAGNETIZED TOROIDAL PLASMAS}

Consider now the formalism developed in Sec. II in application to magnetized plasmas. Assume that a plasma has a toroidal geometry, so that inhomogeneities along the magnetic field are smoothed out and the plasma becomes uniform along a flux surface on time scales large compared to the period of particle rotation along the torus. Similarly, assume uniform distribution over gyrophases, plus assume homogeneous plasma profile across flux surfaces.

Suppose now that the plasma, having an initial distribution $f\left(\mathbf{r}_{1}, \mathbf{p}_{1}\right)$, undergoes Hamiltonian interaction with an electromagnetic field for a limited time, which eventually results in bringing the plasma to some final state $f\left(\mathbf{r}_{2}, \mathbf{p}_{2}\right)$. The number of particles within each phase space element is conserved:

$$
f\left(\Gamma_{1}\right) d \Gamma_{1}=f\left(\Gamma_{2}\right) d \Gamma_{2}
$$

as well as conserved is the distribution function itself, as follows from the Liouville theorem. Assuming spatially uniform both initial and final distributions and neglecting the dependence on the gyrophase, obtain

$$
f\left(\epsilon_{2}, u_{2}\right)=f\left(\epsilon_{1}, u_{1}\right)
$$

where $\epsilon$ is the energy of the particle motion transverse to the dc magnetic field, and $u$ is the particle velocity along the field. Since $d \Gamma_{i}=m^{2} d u_{i} d \epsilon_{i} d \theta_{i} d V_{i}$, where $\theta_{i}$ is the gyrophase and $d V_{i}$ is the element of a spatial volume, from Eqs. (34), (35) one has

$$
d u_{2} d \epsilon_{2}=d u_{1} d \epsilon_{1}
$$

after integrating over $\theta$ and $V$. Eq. (36) can be considered as the requirement of space conservation on an effective phase plane $(u, \epsilon)$, whose evolution can hence be considered a Hamiltonian process with a single degree of freedom $(N=1)$.

A variational formalism, like in Sec. II, can be readily restated for the reduced system. Hence, the absolute maximum and the absolute minimum of the functional

$$
G=\int \psi\left(u_{2}, \epsilon_{2}\right) \phi\left(u_{1}, \epsilon_{1}\right) d \tilde{\Gamma}
$$

$\left(d \tilde{\Gamma} \equiv d \tilde{\Gamma}_{1}=d \tilde{\Gamma}_{2}, d \tilde{\Gamma}_{i}=d u_{i} d \epsilon_{i}\right)$ are realized by phase space transformations, which map the surfaces of constant $\phi$ on the plane $\left(u_{1}, \epsilon_{1}\right)$ to the surfaces of constant $\psi$ on the plane $\left(u_{2}, \epsilon_{2}\right)$ and provide that $\phi(\psi)$ becomes a monotonic function given by Eq. (16). If $d \phi / d \psi \geq 0$, the absolute maximum is realized; if $d \phi / d \psi \leq 0$, then the absolute minimum is obtained. 


\section{CONCLUSIONS}

We developed a variational formalism to study the phase space limitations on the Hamiltonian interaction between plasmas and electromagnetic fields. The solution of the so-called Gardner's restacking problem [1] was given a precise mathematical formulation over a class of piecewise continuous phase space transformations. The analysis was extended to the conditional restacking problem, through which we found the minimum energy state of a plasma with a given current. We also showed how the formalism could be applied to toroidal plasmas in a dc magnetic field.

\section{ACKNOWLEDGMENTS}

The work is supported by DOE contract DE-AC0276-CHO3073.

[1] C. S. Gardner, Phys. Fluids B 6, 839 (1963).

[2] I. B. Bernstein, Phys. Rev. 109, 10 (1958).

[3] N. J. Fisch and J. M. Rax, Phys. Fluids B 5, 1754 (1993).

[4] N. J. Fisch and J. M. Rax, Phys. Rev. Lett. 69, 612 (1992).

[5] I. Y. Dodin and N. J. Fisch, Ponderomotive barrier as a Maxwell demon, submitted to Phys. Plasmas.

[6] N. J. Fisch, J. M. Rax, and I. Y. Dodin, Phys. Rev. Lett. 91, 205004 (2003).

[7] H. Ye and P. J. Morrison, Phys. Fluids B 4, 771 (1992).

[8] N. J. Fisch, Rev. Mod. Phys. 59, 175 (1987). 


\section{External Distribution}

Plasma Research Laboratory, Australian National University, Australia

Professor I.R. Jones, Flinders University, Australia

Professor João Canalle, Instituto de Fisica DEQ/IF - UERJ, Brazil

Mr. Gerson O. Ludwig, Instituto Nacional de Pesquisas, Brazil

Dr. P.H. Sakanaka, Instituto Fisica, Brazil

The Librarian, Culham Laboratory, England

Mrs. S.A. Hutchinson, JET Library, England

Professor M.N. Bussac, Ecole Polytechnique, France

Librarian, Max-Planck-Institut für Plasmaphysik, Germany

Jolan Moldvai, Reports Library, Hungarian Academy of Sciences, Central Research Institute for Physics, Hungary

Dr. P. Kaw, Institute for Plasma Research, India

Ms. P.J. Pathak, Librarian, Institute for Plasma Research, India

Ms. Clelia De Palo, Associazione EURATOM-ENEA, Italy

Dr. G. Grosso, Instituto di Fisica del Plasma, Italy

Librarian, Naka Fusion Research Establishment, JAERI, Japan

Library, Laboratory for Complex Energy Processes, Institute for Advanced Study, Kyoto University, Japan

Research Information Center, National Institute for Fusion Science, Japan

Dr. O. Mitarai, Kyushu Tokai University, Japan

Dr. Jiangang Li, Institute of Plasma Physics, Chinese Academy of Sciences, People's Republic of China

Professor Yuping Huo, School of Physical Science and Technology, People's Republic of China

Library, Academia Sinica, Institute of Plasma Physics, People's Republic of China

Librarian, Institute of Physics, Chinese Academy of Sciences, People's Republic of China

Dr. S. Mirnov, TRINITI, Troitsk, Russian Federation, Russia

Dr. V.S. Strelkov, Kurchatov Institute, Russian Federation, Russia

Professor Peter Lukac, Katedra Fyziky Plazmy MFF UK, Mlynska dolina F-2, Komenskeho Univerzita, SK-842 15 Bratislava, Slovakia

Dr. G.S. Lee, Korea Basic Science Institute, South Korea

Institute for Plasma Research, University of Maryland, USA

Librarian, Fusion Energy Division, Oak Ridge National Laboratory, USA

Librarian, Institute of Fusion Studies, University of Texas, USA

Librarian, Magnetic Fusion Program, Lawrence Livermore National Laboratory, USA

Library, General Atomics, USA

Plasma Physics Group, Fusion Energy Research Program, University of California at San Diego, USA

Plasma Physics Library, Columbia University, USA

Alkesh Punjabi, Center for Fusion Research and Training, Hampton University, USA

Dr. W.M. Stacey, Fusion Research Center, Georgia Institute of Technology, USA

Dr. John Willis, U.S. Department of Energy, Office of Fusion Energy Sciences, USA

Mr. Paul H. Wright, Indianapolis, Indiana, USA 
The Princeton Plasma Physics Laboratory is operated by Princeton University under contract with the U.S. Department of Energy.

\author{
Information Services \\ Princeton Plasma Physics Laboratory \\ P.O. Box 451 \\ Princeton, NJ 08543
}

Phone: 609-243-2750

Fax: 609-243-2751

e-mail: pppl_info@pppl.gov

Internet Address: http://www.pppl.gov 